\title{
The impact of morning light intensity and environmental temperature on body temperatures and alertness
}

Citation for published version (APA):

te Kulve, M., Schlangen, L. J. M., Schellen, L., Frijns, A. J. H., \& van Marken Lichtenbelt, W. D. (2017). The impact of morning light intensity and environmental temperature on body temperatures and alertness. Physiology \& Behavior, 175, 72-81. https://doi.org/10.1016/j.physbeh.2017.03.043

Document status and date:

Published: 01/06/2017

DOI:

10.1016/j.physbeh.2017.03.043

Document Version:

Publisher's PDF, also known as Version of record

\section{Document license:}

Taverne

\section{Please check the document version of this publication:}

- A submitted manuscript is the version of the article upon submission and before peer-review. There can be important differences between the submitted version and the official published version of record.

People interested in the research are advised to contact the author for the final version of the publication, or visit the DOI to the publisher's website.

- The final author version and the galley proof are versions of the publication after peer review.

- The final published version features the final layout of the paper including the volume, issue and page numbers.

Link to publication

\footnotetext{
General rights rights.

- You may freely distribute the URL identifying the publication in the public portal. please follow below link for the End User Agreement:

www.umlib.nl/taverne-license

Take down policy

If you believe that this document breaches copyright please contact us at:

repository@maastrichtuniversity.nl

providing details and we will investigate your claim.
}

Copyright and moral rights for the publications made accessible in the public portal are retained by the authors and/or other copyright owners and it is a condition of accessing publications that users recognise and abide by the legal requirements associated with these

- Users may download and print one copy of any publication from the public portal for the purpose of private study or research.

- You may not further distribute the material or use it for any profit-making activity or commercial gain

If the publication is distributed under the terms of Article $25 \mathrm{fa}$ of the Dutch Copyright Act, indicated by the "Taverne" license above, 


\title{
The impact of morning light intensity and environmental temperature on body temperatures and alertness
}

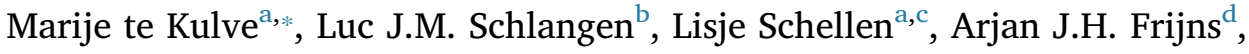 \\ Wouter D. van Marken Lichtenbelt ${ }^{\mathrm{a}}$ \\ a Department of Human Biology \& Movement Sciences, NUTRIM, Maastricht University, Maastricht, The Netherlands \\ b Philips Lighting Research, Eindhoven, The Netherlands \\ ' School of Built Environment and Infrastructure, Avans University of Applied Sciences, Tilburg, The Netherlands \\ d Department of Mechanical Engineering, Eindhoven University of Technology, The Netherlands
}

\section{A R T I C L E I N F O}

\section{Keywords:}

Light

Thermophysiology

Alertness

Sleepiness

Productivity

\begin{abstract}
A B S T R A C T
Indoor temperature and light exposure are known to affect body temperature, productivity and alertness of building occupants. However, not much is known about the interaction between light and temperature exposure and the relationship between morning light induced alertness and its effect on body temperature. Light intensity and room temperature during morning office hours were investigated under strictly controlled conditions. In a randomized crossover study, two white light conditions ( $4000 \mathrm{~K}$, either bright $1200 \mathrm{~lx}$ or dim $5 \mathrm{~lx}$ ) under three different room temperatures $\left(26,29\right.$ and $\left.32{ }^{\circ} \mathrm{C}\right)$ were investigated. A lower room temperature increased the core body temperature (CBT) and lowered skin temperature and the distal-proximal temperature gradient (DPG). Moreover, a lower room temperature reduced the subjective sleepiness and reaction time on an auditory psychomotor vigilance task (PVT), irrespective of the light condition. Interestingly, the morning bright light exposure did affect thermophysiological parameters, i.e. it decreased plasma cortisol, CBT and proximal skin temperature and increased the DPG, irrespective of the room temperature. During the bright light session, subjective sleepiness decreased irrespective of the room temperature. However, the change in sleepiness due to the light exposure was not related to these physiological changes.
\end{abstract}

\section{Introduction}

Light and temperature exposure can increase our alertness during the day and sleepiness in the evening and night. Many temperature and light studies have been carried out in search for optimal conditions. However, most studies tested light and temperature effects separately while the interaction between ambient temperature and lighting conditions may result in a larger acceptance range for one of the conditions without performance decrements. For example, bright light may be able to (partly) mitigate the performance decrements that can occur under more elevated ambient temperatures. It is demonstrated that the intensity, wavelength and duration of light exposure affect alertness and performance during cognitive tasks [1,2]. Experiments on only the effects of light, show that daytime bright light decreases reaction time and sleepiness [3]. Additionally, experiments in a simulated office environment show that the addition of the task lighting improved performance as compared to ceiling luminaries only [4]. During a laboratory study it was found that bright light (2000 lx) improves alertness and productivity during the night and has a positive effect on mood during the day compared to dim light [5]. Studies on the relation between indoor temperature and office tasks productivity revealed that there is an room temperature for which performance of building occupants is highest [6]. Additionally, a higher thermal satisfaction is associated with improved productivity and mood $[7,8]$. Romeijn et al. (2012) also found an optimum to obtain the highest alertness, which is at lower skin temperatures compared to the optimum for sleepiness [9].

A larger distal-proximal skin temperature gradient (DPG) in the evening is associated with a higher sleepiness. Experiments show that evening light can delay the natural decline in core body temperature (CBT), proximal skin temperature and the natural increase in DPG along with a reduced sleepiness [10]. Yet, little is known about the effects of light intensity on thermophysiology at different times of day and its relation to alertness. Contrary to our expectations, a few studies indicate that bright light exposure in the morning decreases the CBT $[11,12]$. Ruger et al. (2006) found no effect of afternoon bright light on

\footnotetext{
* Corresponding author.

E-mail address: m.tekulve@maastrichtuniversity.nl (M. te Kulve).
} 
CBT, but bright light reduced sleepiness [13]. Due to the absent of physiological changes, they suggest that the effect on sleepiness must be caused by an alternative mechanism or pathway which immediately influences alertness in humans. Although light can affect body temperatures, it remains unknown how light history, timing, duration, intensity and spectrum of a light exposure impact thermophysiology [14].

The main objective is to test the effects of morning light intensity and temperature condition on alertness and body temperature. We hypothesise that bright light reduces sleepiness and improves task performance independently of the temperature exposure. We expect that during the morning bright light results in a faster increase of CBT and faster decrease of the DPG due to its suppressive effect on melatonin production. Secondly we hypothesise that a relatively high temperature increase sleepiness and reduces task performance along with a higher means skin temperature and a higher DPG. The second objective is to investigate whether morning light induced alertness changes coincide with changes in body temperature (distribution).

\section{Method}

The Medical Ethical Committee of Maastricht University Medical Centre + approved the study protocol. All participants provided a written informed consent prior to the experiments. All procedures were conducted in accordance with the principles of the Declaration of Helsinki.

\subsection{Participants}

Nineteen healthy female participants took part in the study. Participants were recruited by advertisements on local billboards at the university and at the website www.digi-prik.nl. Participants were screened to meet the following inclusion criteria: Caucasian, generally healthy, age 18 to 30 years, BMI $18-25 \mathrm{~kg} / \mathrm{m}^{2}$, using microgynon 30 or any other anti contraceptive pill consisting of a levonorgestrel/ethinylestradiol combination, and a normal chronotype (Table 1). Exclusion criteria consisted of: colour blindness, ocular pathologies, medication use, pregnancy, hypertension, general feeling of illness at day of experiment, (history of) cardiovascular diseases, contraindication of the telemetric pill, and employees of our research group. Participants were screened by means of a medical questionnaire and a chronotype questionnaire [15].

\subsection{Protocol}

In a randomized crossover design all participants took part in two identical laboratory sessions that only differed in lighting exposure; one session occurred under dim light (5 lx) and the other under bright light (1200 lx) (Fig. 1). The order of the sessions was randomized among participants. The time between on participants' two experimental sessions was 1 or 2 weeks. Both sessions started with baseline exposure at a thermo neutral ambient temperature $\left(29^{\circ} \mathrm{C}\right)$ and a moderate light intensity of $250 \mathrm{~lx}$ and $4000 \mathrm{~K}$ measured in the outward direction of the optical axis at the outer surface of the volunteers eye in its most usual viewing direction. After the baseline, participants were exposed to a

Table 1

Participant characteristics.

\begin{tabular}{ll}
\hline Characteristic & Average $( \pm \mathrm{SD})$ \\
\hline Age $(\mathrm{yr})$ & $22.3 \pm 1.9$ \\
Body mass $(\mathrm{kg})$ & $62.7 \pm 5.5$ \\
Height $(\mathrm{m})$ & $1.70 \pm 0.07$ \\
BMI $\left(\mathrm{kg} / \mathrm{m}^{2}\right)$ & $21.7 \pm 1.8$ \\
Body fat $(\%)$ & $30.2 \pm 3.2$ \\
\hline
\end{tabular}

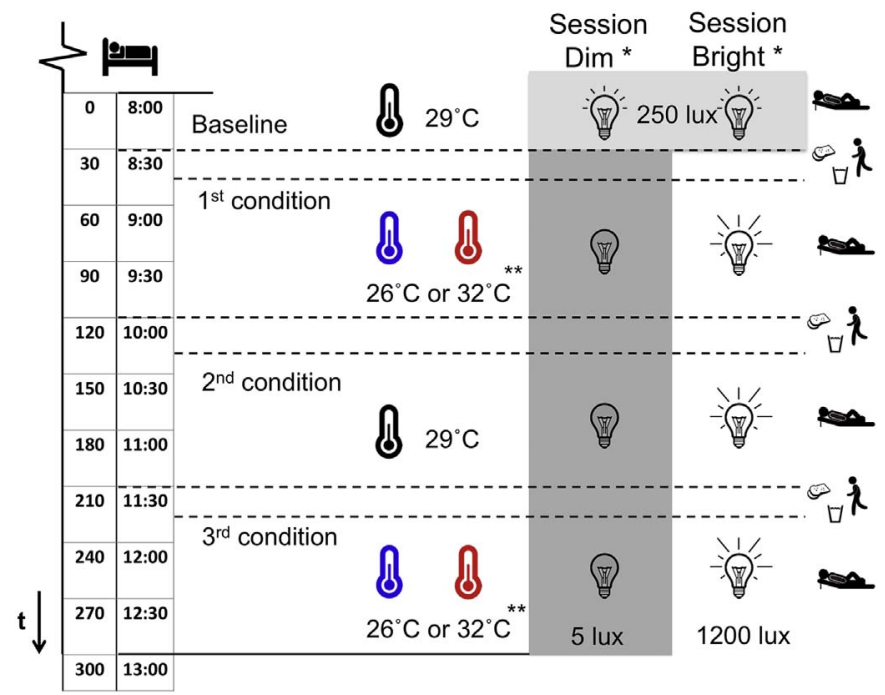

Fig. 1. Schedule of the experiments. *The order of the sessions was randomized among participants; half of them started with the dim light session and the others with the bright light session. **The order of the temperature was randomized among participants, but remained identical between the two sessions of each participant.

series of three ambient temperatures (cool, $26^{\circ} \mathrm{C}$; thermo-neutral, $29^{\circ} \mathrm{C}$; and warm, $32{ }^{\circ} \mathrm{C}$ ), either under bright light or under dim light. The order of the cool and warm condition was randomized across participants; after baseline half of the participants started with the warm condition and ended with the cool condition, the other half started with the cool condition, ending with the warm condition. The thermo-neutral condition was always used as the second condition, to ensure that the magnitude of the change in ambient temperature between the conditions was equal. For each participant, the order of the temperature conditions was identical in both light sessions.

A week prior to the experiments the participant kept an evening and morning sleep diary (see Section 2.3 Measurements for details). All participants took the anti contraceptive pill during the days of the experiments. The evening before the experiments, the participants arrived at the university at 9:00 PM. They refrained from food, caffeine and alcoholic consumption $12 \mathrm{~h}$ before the experiment started. In the evening, the Psychomotor Vigilance Task (PVT) and the questionnaires were trained. The participants also swallowed a capsule to measure CBT (see Measurements Section 2.3.5 for details). They slept in a respiration/climate chamber with a constant indoor ambient temperature of $21{ }^{\circ} \mathrm{C}$, between $11.00 \mathrm{PM}$ and $7.00 \mathrm{AM}$ participants were in dim light (i.e., the lighting was switched off $(<1 \mathrm{~lx})$ ). During the night they wore an actiwatch to monitor sleep. About $15 \mathrm{~min}$ after waking up, the participant ate a small, standardized breakfast (cracker $(53 \mathrm{kcal})$ and water). After that, skin temperature loggers, and devices for measuring CBT were attached and a venous catheter was placed in the antecubital vein. Participants were clothed in underwear $(0.04 \mathrm{clo})$. The preparations between 7:30 and 8:00 AM were done under baseline lighting conditions $(250 \mathrm{~lx})$. Subsequently, the participant entered the respiration chamber for the start of the baseline measurements at 8:00 AM. Here, the skin blood flow probes were attached to the ventral surface of the hand and the underarm. Finally a mask of the indirect calorimetry meter was put over the mouth and nose. The blood pressure monitor was placed on the arm without the catheter.

Once the participants were lying in semi-supine position, the experiment started with a 30-minute lasting baseline with lighting at $250 \mathrm{~lx}$ and a thermo-neutral room temperature $\left(29^{\circ} \mathrm{C}\right)$. During the experiment participants filled out questionnaires about (e.g.) self assessed sleepiness (KSS) every $15 \mathrm{~min}$ and at the end of the baseline $(t=25 \mathrm{~min}) 40 \mathrm{ml}$ blood was drawn. After $10 \mathrm{~min}$ blood pressure was measured and at the end of the baseline $(t=25 \mathrm{~min}) 40 \mathrm{ml}$ blood was drawn. After the $30 \mathrm{~min}$ baseline, the participant moved to an adjacent 


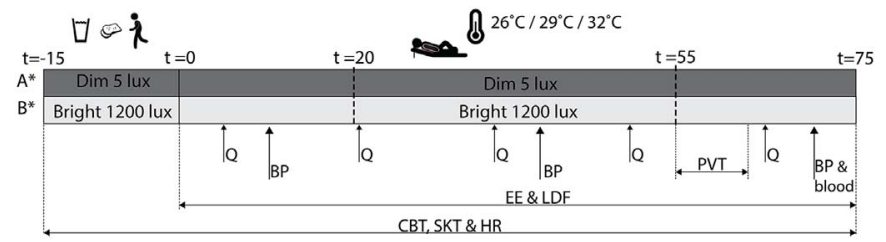

Fig. 2. Procedure during each temperature condition ( $75 \mathrm{~min}$ ). Measurements indicated in this figure are core body temperature (CBT), Skin temperature (SKT), Heart rate (HR), Energy expenditure (EE), Laser Dopler Flowmetry (LDF), Blood pressure (BP), Questionnaires (Q), Alertness task (PVT) and drawing blood from the catheter (blood).

room with a cool $\left(26^{\circ} \mathrm{C}\right)$ or a warm $\left(32^{\circ} \mathrm{C}\right)$ ambient temperature. This room either had dim light (5 lx) or bright light (1200 lx) LED lighting, both with a correlated colour temperature of $4000 \mathrm{~K}$. This protocol is summarized in Fig. 1. During the first $20 \mathrm{~min}$ of each 75 -minute condition the participant got familiarized with the new temperature condition and this time interval was not used in the data analyses. The intervals between the questionnaires was $15 \mathrm{~min}$ and blood pressure was measured every $40 \mathrm{~min}$ starting at $t=10 \mathrm{~min}$ (Fig. 2). A reaction time task test (see Measurements) was performed near the end of each temperature condition $(t=55 \mathrm{~min}$ ). At each temperature condition skin temperature, core temperature, heart rate, energy expenditure and skin blood flow were measured continuously with a 1-minute interval. Each time the participant changed to another temperature condition (and room), there was a small break of 15 min. During this break the temperature and light were identical as in the past condition, and the participant performed standardized stretch exercises and ate a cracker (53 kcal) and was allowed to drink water. After that the participant went to another room with a different temperature.

\subsection{Measurements}

\subsubsection{Indoor environment}

Air temperature and relative humidity were measured at oneminute intervals by means of four dataloggers (iButton, DS1923, Maxim). The iButtons were placed next to the participant at a height of $0.1 \mathrm{~m}, 0.3 \mathrm{~m}, 0.6 \mathrm{~m}$ and $1.1 \mathrm{~m}$. The installed light system was a LED wall washing (Philips SkyRibbon IntelliHue Wall Washing Powercore). The lighting intensity was confirmed with a lux meter (Testo 545) each time the lighting condition changed. The spectrum of each lighting condition was measured once using a radiospectrometer (Jeti).

\subsubsection{Body \& skin temperatures}

Core temperature was measured using a telemetric temperature pill (VitalSense $^{\circledast}$ medical grade capsules, Equivital ${ }^{\mathrm{TM}}$, Hidalgo Limited). The pill was ingested in the evening before the experiment to ensure that it was it was sufficient deep in the intestinal track. Skin temperatures were measured by iButton dataloggers (DS1922L, Maxim) [16]. They were attached to 26 body sites (Fig. 3). Mean skin temperature was calculated using skin temperatures measured at the 14 ISO-defined skin sites [17]. Extra buttons were placed to obtain symmetrical skin temperature data and buttons were placed at the under arm and the middle finger to assess skin temperature gradients.

\subsubsection{Blood parameters}

Blood was collected via a venous catheter in an antecubital vein. The catheter was placed at least 15 min before the baseline measurement. During every blood drawing procedure a total of $40 \mathrm{~mL}$ was taken. For each session, blood was drawn after 25 min during baseline, at 115 min during the 1 st temperature condition, at 205 min during 2 nd temperature condition and at $295 \mathrm{~min}$ during the final temperature condition. The blood samples of the baseline and the 3rd temperature condition were used to determine the change in catecholamine levels during one session [33]. Cortisol levels were measured for all conditions (using Electro Chemi Luminescence Immuno Assay (ECLIA)). Finally

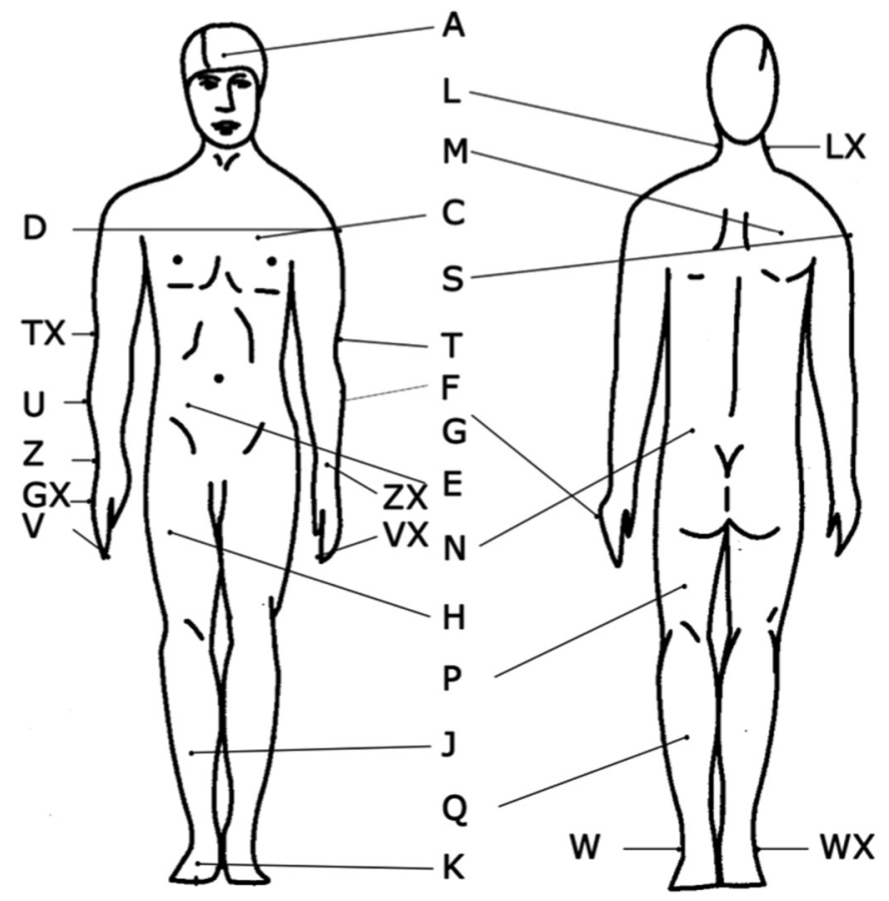

Fig. 3. Body positions of the 26 iButtons.

the blood samples of the baseline condition were used for melatonin analyses. Baseline melatonin levels were determined (Melatonin direct Serum/Plasma/Saliva RIA, IBL international) to verify whether the levels were indeed low (as expected) at the onset of both experimental light conditions.

\subsubsection{Sleepiness \& reaction times}

Every time the thermal questionnaire was administered, also the Karolinska Sleepiness scale (KSS) [18] was used to assess subjective sleepiness/alertness. Reaction time performance was tested using an auditory Psychomotor Vigilance Task (PVT) that lasted $8 \mathrm{~min}$ [19]. During this task the reaction time of the participant to an auditory stimulus was measured. The interstimulus interval was between 2 and $10 \mathrm{~s}$.

\subsubsection{Sleep characteristics}

The sleep characteristics of each participant were measured via a sleep diary (during 1 week prior to the experiments) and the actigraphy (using a Actiwatch Spectrum Philips Respironics) during the night before the experiment. The sleep diary consisted of an evening questionnaire about activities and sleepiness during the day and a morning questionnaire about sleep timing and quality. The average time participants went to bed, woke up and the sleep duration the week prior to the experiment was calculated for each subject (Table 2). During the night at the university, participants wore an Actiwatch Spectrum (Philips Respironics) to measure sleep. The hours of sleep measured by the Actiwatch was calculated as well as the percentage awake (Table 2).

\subsection{Data analyses}

The ambient temperature and relative humidity were averaged for each light session and temperature condition separately; resulting in a total of 8 averages (two light sessions for three temperature conditions and two baselines). Mean body temperatures were calculated over the last $5 \mathrm{~min}$ before the PVT (i.e., between $t=50$ and $t=55 \mathrm{~min}$ ) to obtain the maximal effect of the light and temperature exposure without potential disturbance by the activity of performing the PVT. Energy expenditure, skin blood perfusion and heart rate were averaged 
Table 2

Sleep characteristics of the participants.

\begin{tabular}{|c|c|c|c|c|c|}
\hline & \multicolumn{2}{|c|}{ Actiwatch (night at university) } & \multicolumn{3}{|c|}{ Sleep diary (week prior to experiment) } \\
\hline & Sleep $[\mathrm{h}]$ & Awake [\%] & Bedtime & Wakeup time & Sleep $[\mathrm{h}]$ \\
\hline Lightsession "Bright" & $6.88 \pm 0.52$ & $19 \pm 6$ & $23.56 \pm 0.50$ & $7.98 \pm 0.62$ & $7.85 \pm 0.39$ \\
\hline Lightsession "Dim” & $6.95 \pm 0.39$ & $18 \pm 5$ & $23.60 \pm 0.53$ & $7.96 \pm 0.56$ & $7.76 \pm 0.51$ \\
\hline Significance & $p=0.535$ & $p=0.460$ & $p=0.825$ & $p=0.859$ & $p=0.405$ \\
\hline
\end{tabular}

between $t=20$ and $t=55 \mathrm{~min}$; this is during the time interval from right after familiarization (to the room temperature) to the start of the PVT task. The average result per participant of the questionnaires within the same period (between $t=20$ and $t=55 \mathrm{~min}$ ) was used for analyses (the 2nd, 3rd and 4th questionnaire). For each light and temperature condition the results of the PVT tasks were analyzed by means of four different outcome parameters: the average reaction time, the 10 fastest reaction times, the 10 slowest reaction times and the number of lapses $>0.5 \mathrm{~s}$ [20].

\subsection{Statistical analyses}

A mixed model, repeated over "temperature condition", "light session" and "condition number" with a random intercept was used to test the effect of light, temperature and order (condition number). Backwards selection was used to determine the required fixed factors in the model. The full factorial model had the following structure:

$$
\begin{aligned}
Y= & a+\beta_{1}{ }^{*} \text { light }+\beta_{2}{ }^{*} \text { Temp }_{1}+\beta_{3}{ }^{*} \text { Temp }_{2}+\beta_{4}{ }^{*} \text { CondNr }_{1}+\beta_{5}{ }^{*} \text { CondNr }_{1} \\
& \text { Temp }_{1}+\beta_{6} \text { Light }{ }^{*} \text { Temp }_{1}+\beta_{7} \text { Light }^{*} \text { Temp }_{2}+\beta_{8} \text { Light }^{*} \text { CondNr }_{1} \\
& +\varepsilon_{\text {intercept }}+\varepsilon
\end{aligned}
$$

In the model, the bright light session was used as the reference and set to zero. Dummy variables were created for the temperature conditions. For temperature, the warm condition is used as reference. $\mathrm{Temp}_{1}$ represents the low temperature and $\mathrm{Temp}_{2}$ the thermo-neutral temperature, which is either " 1 " when this conditions is or " 0 ". For the condition numbers, the last condition (3rd) is used as the reference. Since the thermo-neutral temperature is always the 2nd condition, only the 1st condition is compared to the 3rd using the variable "CondNr ${ }_{1}$ " which can be either " 1 " for condition number 1 or " 0 " for condition number 3. The random (participant dependent) intercept is represented by $\varepsilon_{\text {intercept }}$ and the error term is represented by $\varepsilon$.

Melatonin levels at the end of the baseline in the two light sessions were compared using a paired sample $t$-test. A random intercept mixed model was used to test correlations in which all conditions were included (repeated measures). A Pearson correlation was performed when data did not include repeated measures. To analyse the correlations between physiological parameters and subjective votes, mixed models with a random intercept and a random slope was used. All analyses were performed using a two-sided test. Statistical significance was considered for $p$-values $<0.05$. Statistical analyses were performed using IBM SPSS statistics 23.0.

\section{Results}

\subsection{Indoor environmental conditions}

The ambient temperature and relative humidity were not significantly different between the two light sessions (Fig. 4). Lighting intensity was $4 \mathrm{~lx}$ during the dim light conditions, $987 \mathrm{~lx}$ during the bright light condition and $206 \mathrm{~lx}$ during baseline (Table 3). The correlated colour temperature of all light conditions was set at $4000 \mathrm{~K}$ (Table 3 and Fig. 5).

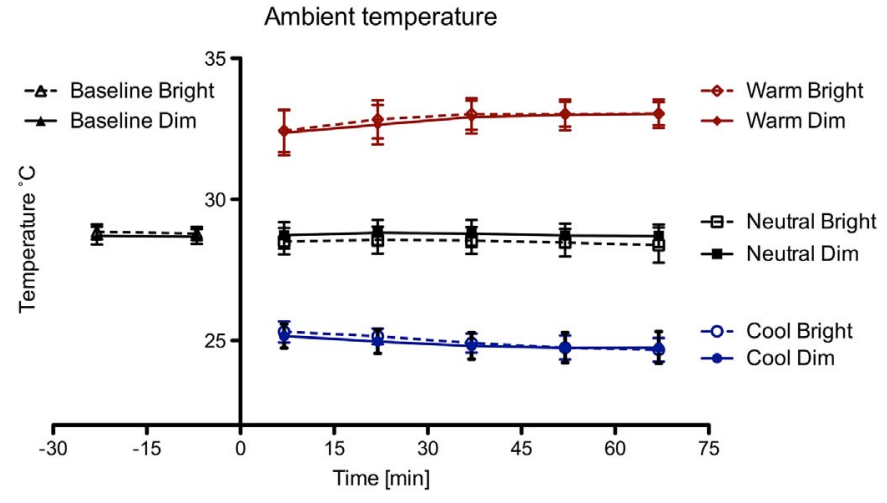

Fig. 4. Measured ambient temperature; average every $15 \mathrm{~min}$ (Average \pm Std. Deviation).

Table 3

Photopic illuminance and the corresponding human retinal photoreceptor weighted "alpha-opic" (equivalent) illuminances calculated according to Lucas et al. [21].

\begin{tabular}{lllll}
\hline & $\lambda_{\max }$ & Bright & Baseline & Dim \\
\hline Photopic lux & 555 & 986.68 & 206.06 & 4.13 \\
"Cyanopic lux" & 419 & 491.44 & 98.25 & 1.77 \\
"Melanopic-lux" & 480 & 762.1 & 154.66 & 2.9 \\
"Rhodopic-lux" & 496.3 & 823.99 & 168.72 & 3.24 \\
"Chloropic-lux" & 530.8 & 895.21 & 185.47 & 3.66 \\
"Erythropic-lux" & 558.4 & 974.23 & 203.38 & 4.07 \\
CCT [K] & & 3776 & 3676 & 3517 \\
\hline
\end{tabular}

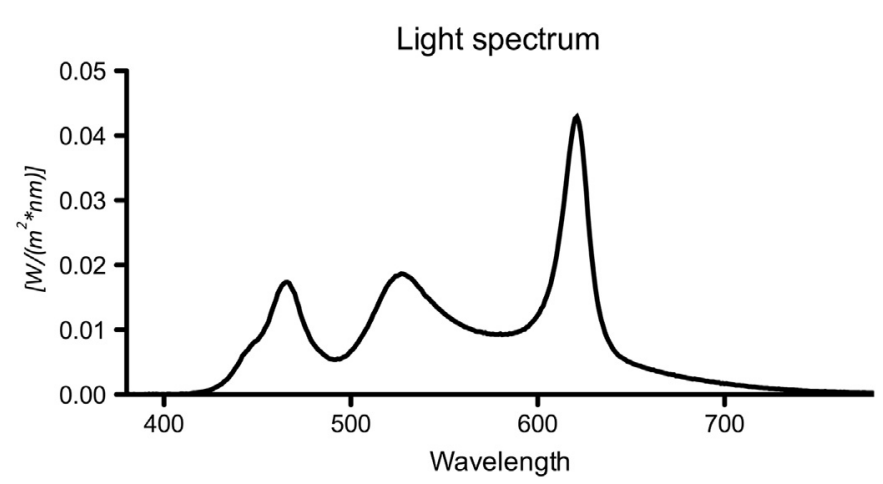

Fig. 5. Spectrum of the LED room lighting with a correlated colour temperature (CCT) of $4000 \mathrm{~K}$ as measured for the bright light setting of $1200 \mathrm{~lx}$.

\subsection{Sleepiness and alertness}

The dim light session resulted in a higher subjective sleepiness compared to the bright light session $(p=0.038)$ (Fig. 6A). However, the average reaction time of the PVT was not influenced by the light intensity $(p=0.305)$ (Fig. 6B). Additionally, the 10 fastest reactions times of the PVT, the 10 slowest reaction times and the amount of lapses (reaction times $>0.5 \mathrm{~s}$ ) on the PVT did not significantly differ between the bright and dim light session. The effects of the light session were independent of the ambient temperature. Sleepiness and the PVT 

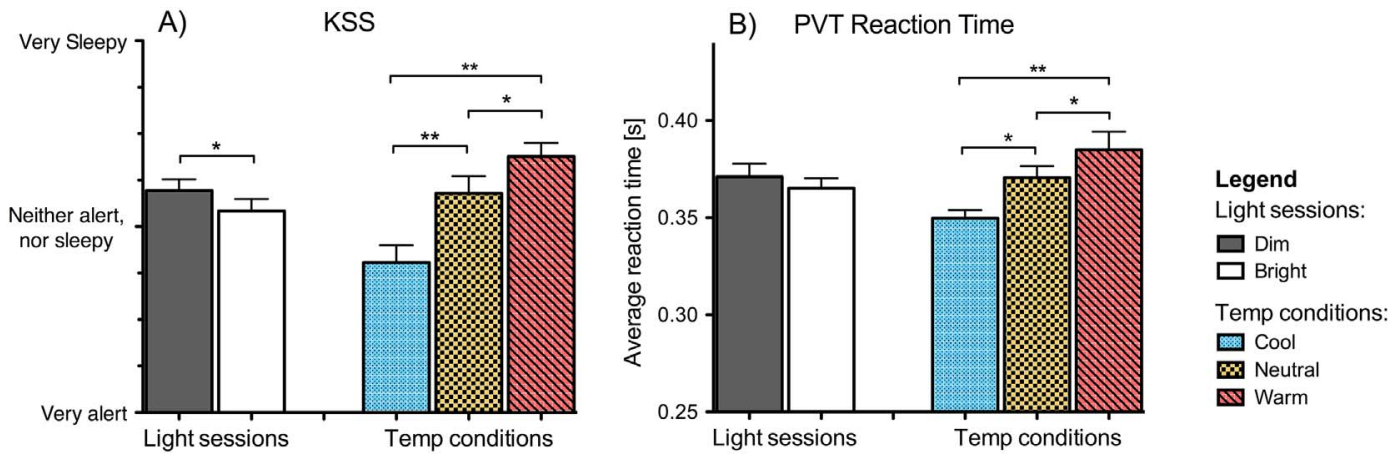

Fig. 6. Sleepiness as rated on the KSS (A) and average reaction time PVT (B), both average \pm SEM and ${ }^{*} p<0.05$ and ${ }^{* *} p<0.01$.

performance were both influenced by the ambient temperature: the highest sleepiness and the slowest reaction times occurred during the warm condition $(p<0.01)$. Subjective sleepiness was also affected by time: participants reported to feel more sleepy during the first temperature condition as compared to the last temperature condition within a light session $(p<0.01)$. There was no significant effect of time on the PVT performance within a light session.
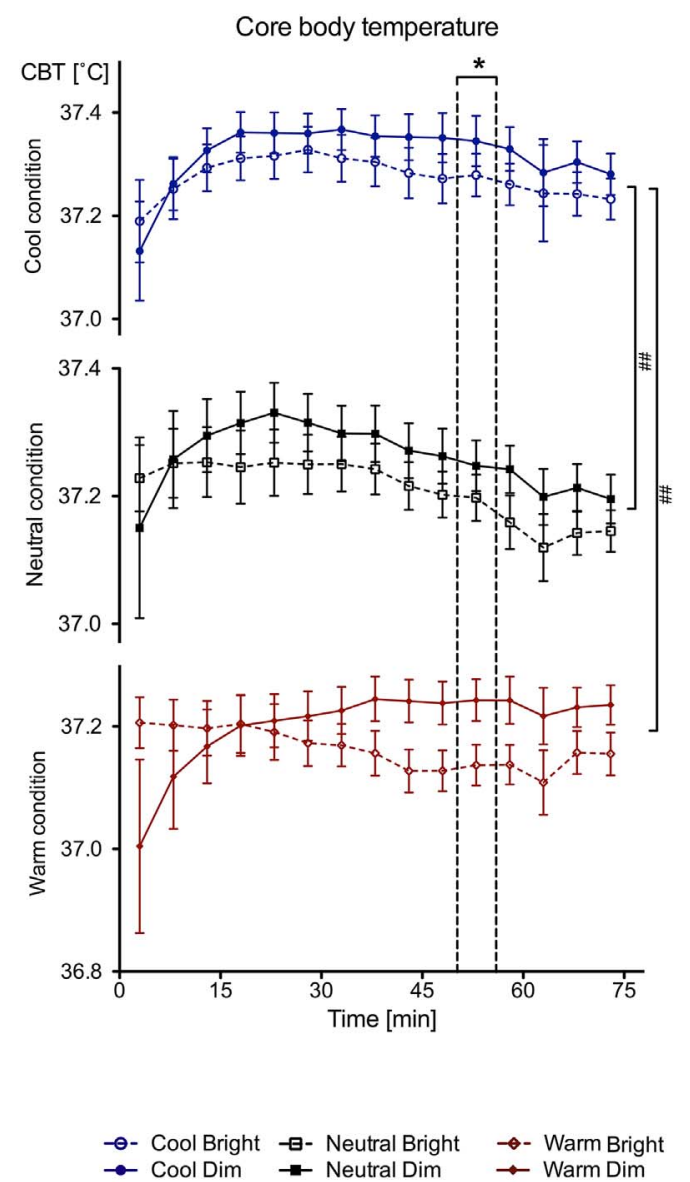

\subsection{Thermophysiology}

Compared to dim light, bright light decreased CBT $\left(\Delta 0.1^{\circ} \mathrm{C}\right.$, $p=0.013)$, decreased proximal skin temperature $\left(\Delta 0.2{ }^{\circ} \mathrm{C}, p<0.01\right)$ and increased the DPG $\left(\Delta 0.3^{\circ} \mathrm{C}, p=0.01\right)$ (Fig. 7$)$, irrespective of the room temperature. As expected, the warm condition resulted in higher mean skin temperature, proximal, distal skin temperatures and DPG compared to thermo-neutral and cool conditions (Fig. 7). The CBT
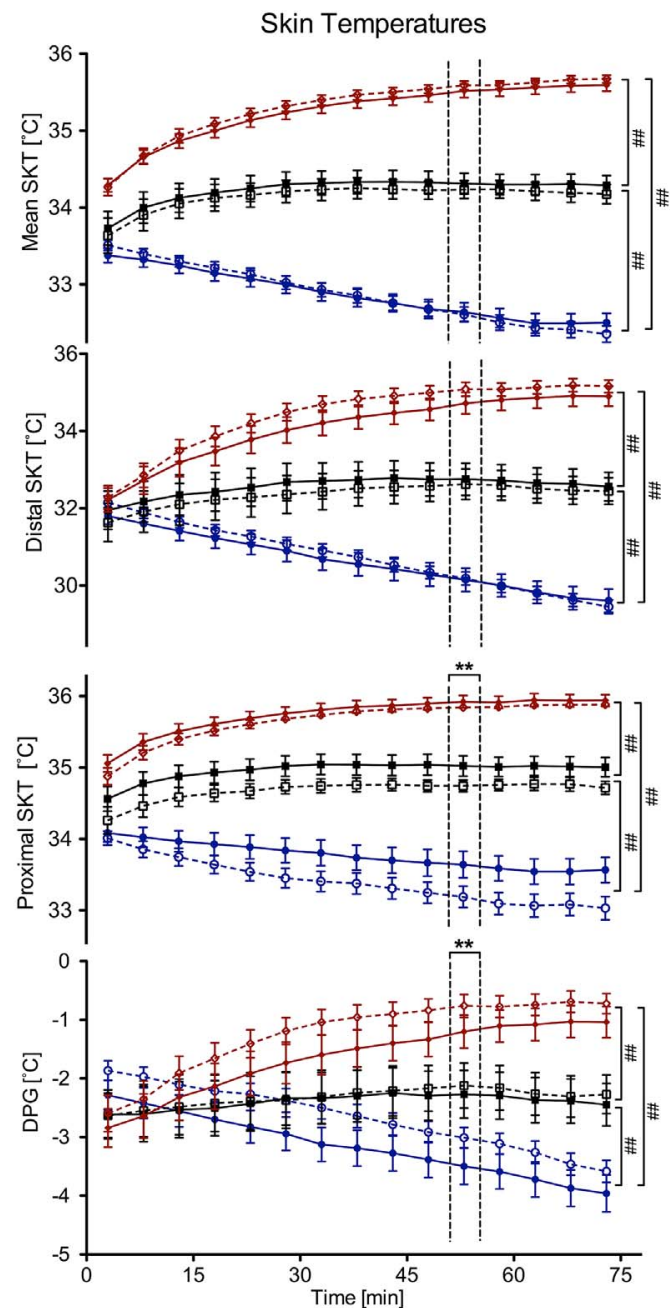

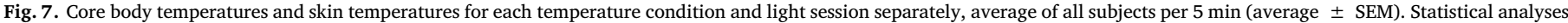

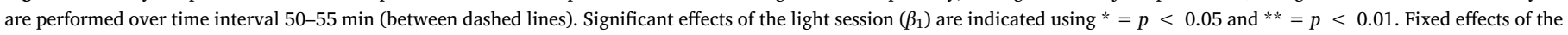
temperature condition $\left(\beta_{2}\right.$ and $\left.\beta_{3}\right)$ are indicated as follows: \# represents $p<0.05$ and \#\# represents $p<0.01$. 
Table 5

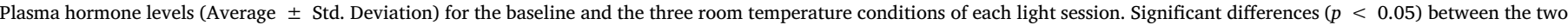
light sessions are indicated in bold.

\begin{tabular}{|c|c|c|c|c|c|c|}
\hline & Light-session & Baseline & 1st temperature condition & 2nd temperature condition & 3rd temperature condition (End) & $\Delta=$ End - baseline \\
\hline \multirow[t]{2}{*}{ Plasma melatonin $[\mathrm{pg} / \mathrm{ml}]$} & Bright & $39.09 \pm 26.47$ & NA & NA & NA & NA \\
\hline & Dim & $43.38 \pm 30.7$ & NA & NA & NA & NA \\
\hline \multirow[t]{2}{*}{ Cortisol $[\mu \mathrm{mol} / \mathrm{l}]$} & Bright & $0.74 \pm 0.16$ & $0.58 \pm 0.16$ & $0.46 \pm 0.14$ & $0.39 \pm 0.15$ & -0.35 \\
\hline & Dim & $0.76 \pm 0.21$ & $0.61 \pm 0.21$ & $0.48 \pm 0.18$ & $0.45 \pm 0.19$ & -0.31 \\
\hline \multirow[t]{2}{*}{ Adrenaline [nmol/l] } & Bright & $0.07 \pm 0.03$ & NA & NA & $0.08 \pm 0.04$ & 0.01 \\
\hline & Dim & $0.06 \pm 0.03$ & NA & NA & $0.10 \pm 0.06$ & 0.04 \\
\hline \multirow[t]{2}{*}{ Noradrenaline [nmol/1] } & Bright & $1.07 \pm 0.30$ & NA & NA & $1.31 \pm 0.41$ & 0.24 \\
\hline & Dim & $1.12 \pm 0.36$ & NA & NA & $1.30 \pm 0.46$ & 0.18 \\
\hline \multirow[t]{2}{*}{ Dopamine [nmol/1] } & Bright & $0.05 \pm 0.02$ & NA & NA & $0.05 \pm 0.02$ & 0.00 \\
\hline & Dim & $0.05 \pm 0.02$ & NA & NA & $0.05 \pm 0.01$ & 0.00 \\
\hline
\end{tabular}

under the warm condition was smaller compared to the thermo-neutral and cool conditions (Fig. 7). The condition number (time) also had an effect on CBT. For example, in all sessions CBT was higher during the last temperature condition as compared to the first temperature condition, independent of the light and temperature conditions. No interactions between the light session and the temperature conditions were found for any of the parameters. An overview of the results is attached in the Appendix Table I. There was no main effect of the light condition on energy expenditure (EE), skin blood perfusion (SBF), heart rate (HR) and blood pressure (BP). Moreover, no significant interactions between the light session and temperature condition were found for these parameters. Details are provided in the Appendix Table II.

\subsection{Blood parameters}

The baseline melatonin levels were not significantly different between the light sessions $(p=0.359)$ (Table 5$)$, while cortisol levels were lower during the bright light as compared to dim light session $(\Delta 0.043 \mu \mathrm{mol} / 1, p=0.041)$ (Table 5 and Fig. 8). Independent of light and temperature, a main effect of time on cortisol was found; the levels declined across the morning $(p<0.01)$. Adrenaline during the light sessions increased compared to baseline. During the dim light session the adrenaline increase was larger than during the bright light session $(\Delta 0.025 \mathrm{nmol} / \mathrm{l}, p=0.035)$ (Table 5$)$. The increases in noradrenaline and dopamine did not differ significantly between the two light sessions ( $p=0.547$ and $p=0.956)$. Noradrenaline was higher during the cool condition compared to warm condition ( $\Delta=0.45 \mathrm{nmol} / 1, p=0.013)$.

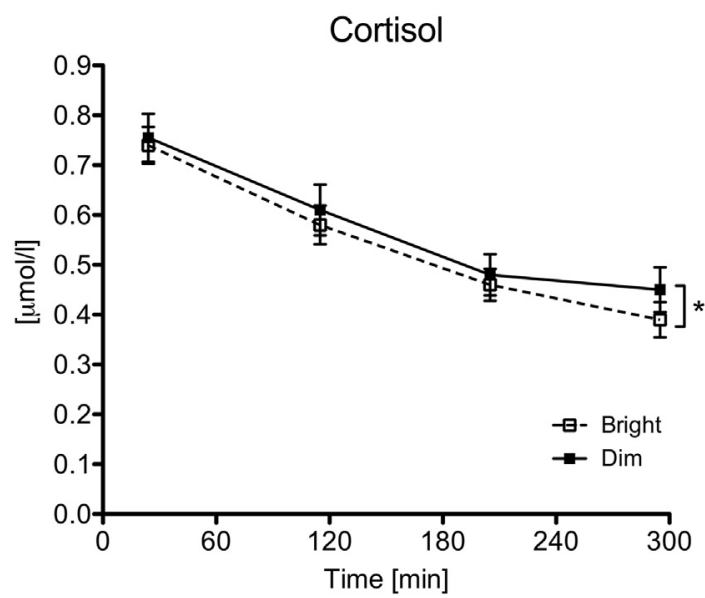

Fig. 8. Plasma cortisol levels during the experiment (at the end of each temperature condition) average $\pm \mathrm{SEM},{ }^{*} p<0.05$ for main effect of light $\left(\beta_{1}\right)$ for the three conditions.
KSS score \& Distal - Proximal Gradient Correlation per subject

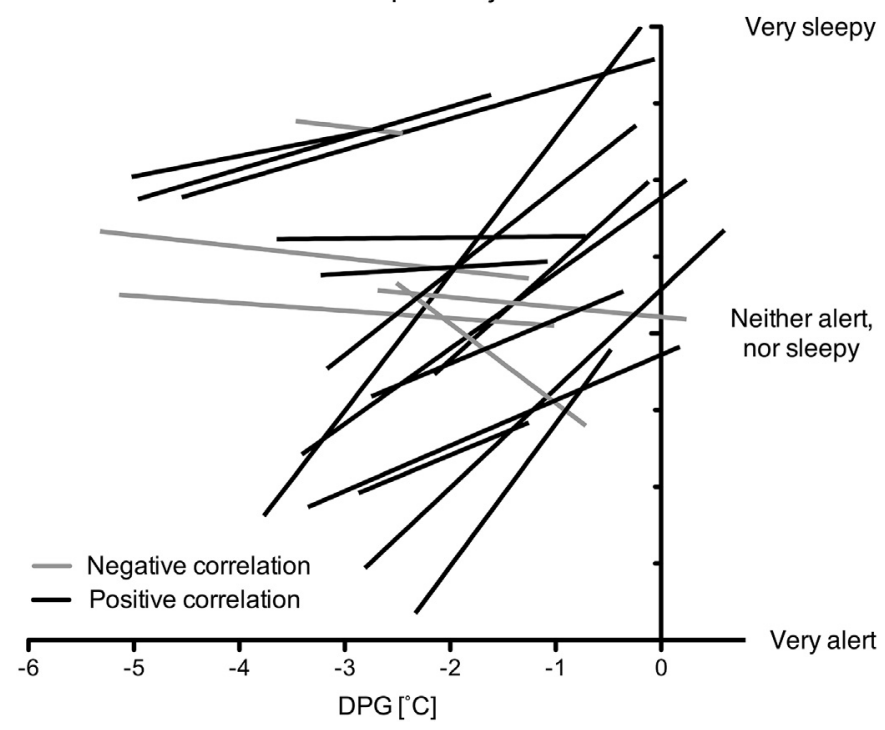

Fig. 9. Within participant correlations between KSS and distal-proximal gradient for all volunteers.

\subsection{Relation body temperatures \& alertness}

We further explored the correlation between the DPG, KSS and PVT. When analysing the whole experiment, taking into account the repeated measures per person, the data show that, overall, the DPG and selfassessed sleepiness (KSS) were significantly positively correlated ( $p=0.015 \& \beta=0.433$ ). A lower DPG (lower room temperature) was generally associated with a lower sleepiness score (see Fig. 9). However, the bright-dim difference $(\Delta)$ in the DPG and the $\Delta$ KSS were not correlated within each individual temperature condition (see Fig. 10) or condition number (time effect). The PVT scores also correlated significantly with the DPG ( $p<0.01$ and $\beta=0.0072)$, but no correlation was observed between the $\triangle \mathrm{PVT}$ or $\triangle \mathrm{KSS}$ and the $\triangle \mathrm{CBT}$ or $\triangle \mathrm{DPG}$. The change in DPG and CBT between the two light sessions was not related to the change in self-assessed sleepiness or reaction time.

\section{Discussion}

This strictly controlled study shows that light intensity significantly affects thermophysiological parameters. Morning bright light exposure decreased CBT and proximal skin temperature and increased the DPG as compared to the dim light condition, irrespective of the room temperature. 


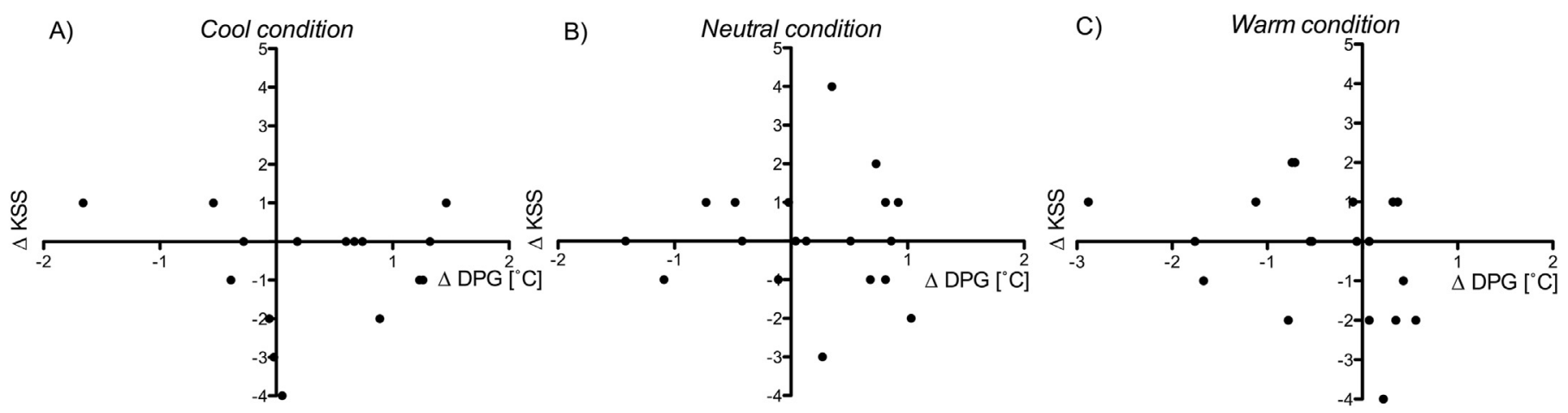

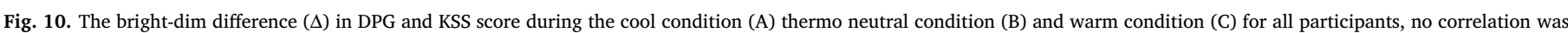
found. $\Delta$ represents the difference between bright and dim session.

\subsection{Effect of temperature on sleepiness, reaction times \& body temperatures}

In line with our expectations, sleepiness during the warm exposure was significantly higher and reaction time significantly slower as compared to the thermo-neutral and cool condition. Lan et al. (2011) tested the effect of temperature in an artificial office environment and also found that a high ambient temperature went along with a reduced performance on reaction time tasks [8]. They hypothesized that the best performance occurs under thermo-neutral conditions, while thermal discomfort (either too cold or too warm) induces performance decrements. According to a study of Romeijn et al., alertness is highest for a skin temperature around $29^{\circ} \mathrm{C}$ [9]. For the range of skin temperatures that we measured (all higher than $29^{\circ} \mathrm{C}$ ), the curve from Romeijn et al. predicts that alertness will reduce when room temperature increases along with higher skin temperatures. Indeed, our PVT results are in good agreement with this prediction. The peak in that alertness curve occurs at a skin temperature well below the values that we measured, so the cool condition in the current study probably was too warm to have a negative effect on performance. However, it should be noted that the comparison between studies may be difficult due to differences in posture and activity. Activity and posture of the participant may influence the optimal room temperature and body temperature for the highest performance and lowest sleepiness.

\subsection{Effect of light on sleepiness and reaction times}

Our results confirm the hypothesis that bright light reduces sleepiness and improves task performance independent of the room temperature. The temperature conditions did not significantly influence the effects of light on self-assessed sleepiness and PVT reaction time. Previous studies also reported a reduced sleepiness under bright light as compared to dim light $[2,3,13]$. In a study with bright light exposure between 12 and $2 \mathrm{PM}$, the reduced sleepiness coincided with a faster PVT reaction time than under dim light [3].

Although we used similar light intensities, the PVT in our study was not influenced by the light exposure. Experiments by Santhi et al. [22] and Badia et al. [23] also did not show an effect of the light intensity on respectively: reaction time (auditory PVT) and computer tasks. Smolders et al. on the other hand, found a positive effect on reaction time of the auditory PVT using bright light (1000 lx) versus light with a low intensity ( $200 \mathrm{~lx}$ ) during the morning but not during the afternoon [24]. Gabel et al. [25] found that dawn simulating light (starting before wake-up), as compared to dim light $(<8 \mathrm{~lx})$, improved performance throughout the day on various tasks (motor tracking, sustained attention to response, working memory, and on a simple reaction time task), while there was no significant effect of dawn simulating light on the PVT task. This is in line with our study in which we did not observe an effect of light exposure on PVT. In addition, the baseline light intensity could have reduced the participants' sensitivity to the subsequent bright light exposure or blunted the effects of dim versus bright.

Finally the time of the year may have affected the results on PVT. Rautkylä et al. reported that a high CCT (correlated colour temperature) improved alertness during autumn but not during spring [26]. Season could change the sensitivity to light by influencing the prior light history, moreover individual differences in light sensitivity can be large [27]. The current study was performed during winter and spring. More research is needed to understand the role of these factors in the heterogeneous results presented in literature and to answer the question whether light exposure is capable of reducing the negative effects of a warm ambient temperature on alertness.

\subsection{Effect of light on thermophysiology}

This study showed that morning light conditions affect human thermophysiology. Under bright light exposure, the CBT was lower as compared to dim light exposure. This is in contrast to our hypotheses stating that the possible phase advancing action of morning bright light on the circadian rhythm, would result in a higher CBT under morning light exposure. The unexpected results could be caused by the relatively high intensity $(200 \mathrm{~lx}$ ) baseline previous to the dim and bright light exposure. However, the decreased CBT under bright light observed in our study is in accordance with results of previous studies $[11,12,28,29]$. Though, in these studies the direct effects of light were not reported and/or the sample sizes were very small $(n=5-9$ participants). Interestingly, the effects of light (intensity) during the evening or night as reported in previous studies, show the opposite effect for what we observed in the morning: in the evening bright light suppresses the natural decline of CBT [30-32].

Our study shows that with respect to skin temperatures proximal skin temperatures were lower and the DPG was higher during bright light conditions. The results of previous studies on the effects of light on skin temperature are heterogeneous. The experiment of Ishimoto et al., showed that forehead skin temperature was lower after bright light (2500 lx) compared to light with a moderate intensity (200 lx) [33]. Two other studies that measured the effect of bright light (3000-5000 lx) on skin temperatures in the morning did not find a significant difference between the light exposures [12,34]. The reasons in this case can be that the bright light exposure was compared to a moderate light intensity (50-150 lx) instead of dim light, or because there was no distinction between proximal and distal skin temperatures $[12,34]$. Notably, bright light in the evening lowers the DPG and the proximal skin temperatures as compared to dim light [10]. This is at least partly attributable to a light induced suppression of the melatonin production $[35,36]$. Melatonin is associated with a lower CBT, a DPG closer to zero, higher distal skin temperatures and higher sleepiness, as demonstrated by Krauchi et al. using exogenous melatonin administration during the afternoon (when melatonin is essentially absent) [37]. In general, melatonin levels drop naturally to very low values within 
$2 \mathrm{~h}$ after rise time [38]. Morning light exposure is expected to hasten the natural morning drop in melatonin levels, thus strengthening the natural circadian increase in alertness and CBT and decrease in DPG. Hence, we would expect a circadian increase in CBT and decrease DPG in the morning, along with a circadian drop in distal skin temperature and sleepiness in the morning. In this study, morning bright light exposure indeed decreased sleepiness, but surprisingly decreased CBT and increased DPG, as compared to dim light. A possible explanation may be found in cortisol levels and increased sympathetic nervous activity.

Firstly, with respect to cortisol we found that cortisol levels were lower for the bright light condition. This is in accordance with results of the study by Jung et al. who reported reductions in plasma cortisol levels after exposure to $6.7-\mathrm{h}$ of bright light $(10,000 \mathrm{~lx})$ [39]. On the contrary, Scheer et al. observed higher cortisol levels while exposing volunteers to darkness and to light of $800 \mathrm{~lx}$ for 1-h during two consecutive mornings [40]. The effects of bright light were only observed at 20 and $40 \mathrm{~min}$ after waking up. Samples analyzed in the current experiment are taken at $90 \mathrm{~min}$ after wake up and probably fall after the cortisol peak in the early morning as supported by the decreasing cortisol levels during the current experiment. This suggests that, similar to the effects on CBT, the timing of light exposure, together with its intensity and duration, may be important in determining how light affects cortisol levels.

Secondly, with respect to the sympathetic nervous activity we found that morning dim light, as compared to bright light, resulted in a larger increase of adrenaline and higher cortisol levels that points towards a higher sympathetic activation [41,42]. Moreover, the findings of Jung et al. (2010) support the existence of a link between photic information and the adrenal glands: they report reduced cortisol levels under bright light exposure [39]. As such, the higher CBT and lower DPG during dim light exposure in the morning might be the result of an increased cortisol level and enhanced sympathetic activation upon a transition from bright(er) light to dim light.

\subsection{Relation body temperatures \& alertness}

In the evening the DPG is positively correlated with sleepiness: a larger DPG (closer to zero) is correlated with a higher sleepiness $[43,44]$. Indeed, in our study population, a higher room temperature resulted in a higher DPG and a higher sleepiness and PVT reaction time as associated with a higher performance [45]. This relation was also present within our room temperature effects on alertness and body temperatures; a higher CBT during the cool condition went along with a reduced self-assessed sleepiness and improved (PVT) reaction times. Surprisingly, morning bright light resulted in a lower CBT and higher DPG while subjective alertness increased. However, the bright light induced increase in DPG and sleepiness are minor as compared to the effects of the room temperature (Fig. 7).

Further analyses on the correlation between the DPG, KSS and PVT showed that the bright-dim difference $(\Delta)$ in the DPG and the $\Delta$ KSS were not correlated within each individual temperature condition. The change in DPG and CBT between the two light sessions was not related to the change in self-assessed sleepiness or reaction time. So the change in sleepiness due to the light exposure was not related to the physiological changes of CBT or DPG. This suggests that the relation between alertness and thermophysiological parameters such as CBT and DPG modulated by light exposure depends on the time of day, and possibly also on the presence or absence of melatonin. This merits further research.

\subsection{Study limitations}

A baseline light intensity of $200 \mathrm{~lx}$ was chosen as an intermediate condition between the dim and bright light session and representative for low intensity indoor lighting. However, the baseline used of $200 \mathrm{~lx}$ instead of dim light may have reduced the sensitivity to subsequent light exposure and could have changed the effect size and/or direction of the physiological responses. Secondly, the timing of the experiments was kept the same for all individuals, independent of their Dim Light Melatonin Onset (DLMO). On the other hand, variation in DLMO was minimized by including normal chronotypes only [15]. Table 2 shows the average and standard deviation of bedtimes of the participants and shows small differences among participants. This means that the circadian timing of the subjects during the experiments was comparable, but nevertheless circadian timing might have influenced the results. Finally, posture and previous temperature condition may have affected results in the subsequent condition. To take the temperature history into account the order of the temperature conditions was randomized among subject but kept the same within subjects.

\section{Conclusion}

This tightly controlled laboratory study showed that light intensity (bright vs dim) and room temperature (three conditions: cool, thermoneutral or warm) independently affect body temperatures and alertness in the morning. PVT reaction times were faster during the lower room temperature as compared to the thermo-neutral and high room temperatures. Additionally, self-assessed sleepiness was highest under the warm condition. A reduction in room temperature decreased skin temperature and increased the core body temperature (CBT) and lowered the distal - proximal skin temperature gradient (DPG).

Bright light reduced self-assessed sleepiness compared to dim light, which is in agreement with previous studies on nocturnal light exposure. However, in this study morning bright light resulted in a lower CBT, a lower proximal skin temperature and a higher DPG as compared to dim light. These responses are opposite to those reported in literature for evening and nighttime bright light exposure. This indicates that the effects of light on thermophysiological parameters such as CBT and DPG are time-of-day and circadian-phase dependent.

Our study results confirm that temperature conditions can improve alertness and reaction time, for which physiological parameters such as body and skin temperatures can be used as indicators. However, the effects of light exposure (as used in this study) on body temperatures and reaction time are less pronounced than of the imposed room temperatures.

The fact that morning bright light exposure reduced sleepiness without decreasing the DPG is unexpected and therefore it would be interesting to explore whether the association between sleepiness and DPG is (circadian) time dependent. Profound knowledge on how alertness is affected by the timing and light history is desired, so that light exposure can be used to (partly) compensate reduced alertness caused by a high ambient temperature.

\section{Conflict of interest}

Luc JM Schlangen is an employee of Philips Lighting Research, the Netherlands.

\section{Acknowledgements}

Bob Wolf and Daan Duijf are thanked for their practical work during the experiments. Jan Souman is thanked for his thoughtful comments on the manuscript. Paul Schoffelen and Marc Souren are gratefully acknowledged for their technical assistance. All participants are thanked for their participation. This project was funded by the STW-Philips Electronics Nederland B.V. Partnership Program 'Advanced Sustainable Lighting Solutions' (no. 12733). 


\section{Appendix A}

Table I

Body temperatures.

\begin{tabular}{|c|c|c|c|c|c|c|c|c|}
\hline & & Baseline & Light-session & Cool & Thermo neutral & Warm & 1st condition & 3rd condition \\
\hline \multirow[t]{2}{*}{ CBT } & Bright & $37.2 \pm 0.20$ & $37.2 \pm 0.2$ & $37.3 \pm 0.2$ & $37.2 \pm 0.2$ & $37.2 \pm 0.2$ & $37.2 \pm 0.2$ & $37.3 \pm 0.2$ \\
\hline & Dim & $37.2 \pm 0.17$ & $37.3 \pm 0.2$ & & & & & \\
\hline Statistics & & $p=0.974$ & $p=0.013^{*}$ & \multicolumn{2}{|c|}{$\begin{array}{l}\text { Cool }>\text { neutral } * \text {, cool }>\text { warm } * * \\
\text { neutral }>\text { warm ns }\end{array}$} & & 1 st $<3$ rd $^{* *}$ & \\
\hline \multirow[t]{2}{*}{ Mean SKT } & Bright & $34.1 \pm 0.40$ & $34.2 \pm 1.3$ & $32.6 \pm 0.5$ & $34.3 \pm 0.6$ & $35.6 \pm 0.3$ & $34.3 \pm 1.5$ & $34.1 \pm 1.6$ \\
\hline & Dim & $34.0 \pm 0.33$ & $34.2 \pm 1.3$ & & & & & \\
\hline Statistics & & $p=0.328$ & $p=0.934$ & $\begin{array}{l}\text { Cool }<\text { neut } \\
\text { neutral }<\text { w }\end{array}$ & $\begin{array}{l}\mathrm{l}^{* * *}, \text { cool }<\text { warm }^{* * *} \\
\mathrm{~m}^{* *}\end{array}$ & & $1 \mathrm{st}>3 \mathrm{rd}^{* *}$ & \\
\hline \multirow[t]{2}{*}{ Prox SKT } & Bright & $34.7 \pm 0.40$ & $34.7 \pm 1.2$ & $33.4 \pm 0.8$ & $34.9 \pm 0.5$ & $35.9 \pm 0.3$ & $34.8 \pm 1.2$ & $34.7 \pm 1.5$ \\
\hline & Dim & $34.7 \pm 0.47$ & $34.9 \pm 1.1$ & & & & & \\
\hline Statistics & & $p=0.997$ & $p<0.01$ & $\begin{array}{l}\text { Cool }<\text { neut } \\
\text { neutral }<\text { w }\end{array}$ & $\begin{array}{l}\mathrm{l}^{* * *}, \text { cool }<\text { warm }^{* * *} \\
\mathrm{~m}^{* *}\end{array}$ & & $\begin{array}{l}\text { Cool: } 1 \text { st }> \\
\text { ns }\end{array}$ & , warm: 1 st $<3$ rd \\
\hline \multirow[t]{2}{*}{ Distal SKT } & Bright & $32.9 \pm 0.81$ & $32.8 \pm 2.3$ & $30.3 \pm 1$ & $32.7 \pm 1.8$ & $34.9 \pm 1$ & $33.1 \pm 2.7$ & $32.5 \pm 2.3$ \\
\hline & Dim & $32.5 \pm 1.11$ & $32.7 \pm 2.3$ & & & & & \\
\hline Statistics & & $p=0.202$ & $p=0.644$ & $\begin{array}{l}\text { Cool }<\text { neut } \\
\text { neutral }<\text { w }\end{array}$ & $\begin{array}{l}\mathrm{l}^{* * *}, \text { cool }<\text { warm }^{* * *} \\
\mathrm{~m}^{* *}\end{array}$ & & 1 st $>3 \mathrm{rd}^{* *}$ & \\
\hline \multirow[t]{2}{*}{ DPG } & Bright & $-1.8 \pm 0.90$ & $-1.9 \pm 1.5$ & $-3.1 \pm 1$ & $-2.2 \pm 1.7$ & $-1 \pm 1.1$ & $-1.7 \pm 1.8$ & $-2.2 \pm 1.1$ \\
\hline & Dim & $-2.2 \pm 1.27$ & $-2.2 \pm 1.6$ & & & & & \\
\hline Statistics & & $p=0.253$ & $p=0.010$ & $\begin{array}{l}\text { Cool }>\text { neut } \\
\text { neutral }>\text { w }\end{array}$ & $\begin{array}{l}\text { l**, cool > warm }{ }^{* * *}, \\
\mathrm{~m}^{* *}\end{array}$ & & $\begin{array}{l}\text { Cool: } 1 \text { st }> \\
1 \text { st }<3 \text { rd }^{* * *}\end{array}$ & , Warm: \\
\hline
\end{tabular}

$\# p<0.10$

${ }^{*} p<0.05$.

${ }^{* *} p<0.01$.

Table II

Physiological parameters.

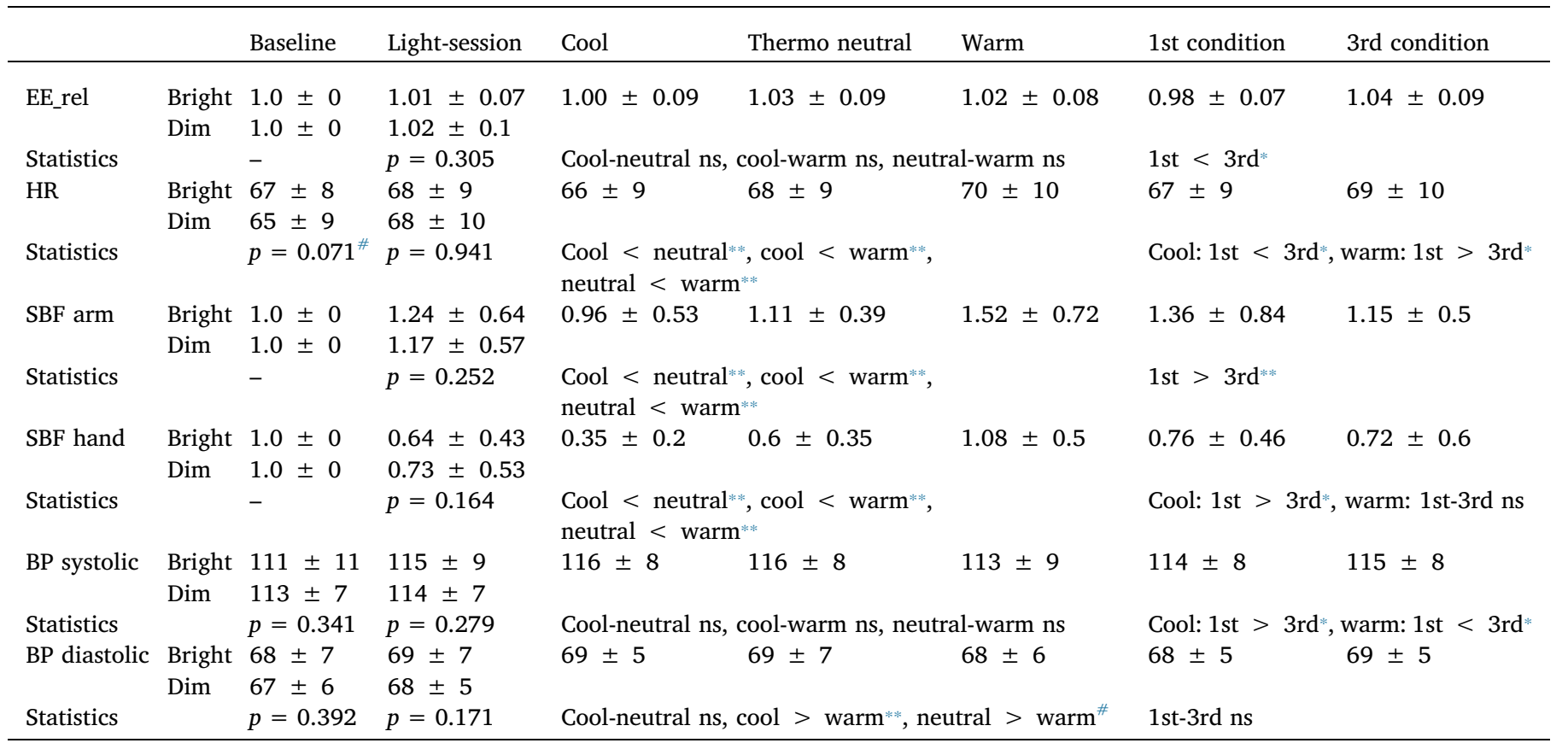

${ }^{\#} p<0.10$.

${ }^{*} p<0.05$.

** $p<0.01$.

\section{References}

[1] G. Vandewalle, P. Maquet, D.J. Dijk, Light as a modulator of cognitive brain function, Trends Cogn. Sci. 13 (10) (2009) 429-438.

[2] C. Cajochen, Alerting effects of light, Sleep Med. Rev. 11 (6) (2007) 453-464.

[3] J. Phipps-Nelson, et al., Daytime exposure to bright light, as compared to dim light, decreases sleepiness and improves psychomotor vigilance performance, Sleep 26 (6) (2003) 695-700.

[4] G. Newsham, et al., Task lighting effects on office worker satisfaction and performance, Leukos 1 (4) (2005) 7-26.

[5] A. Daurat, et al., Bright light affects alertness and performance rhytms during a $24 \mathrm{~h}$ constant routine, Physiol. Behav. 53 (1993) 929-936.

[6] O. Seppanen, W.J. Fisk, Q.H. Lei, Effect of Temperature on Task Performance in 
Office Environment, Lawrence Berkeley National Laboratory, 2006.

[7] S.-I. Tanabe, M. Haneda, N. Nishihara, Workplace productivity and individual thermal satisfaction, Build. Environ. 91 (2015) 42-50.

[8] L. Lan, P. Wargocki, Z. Lian, Quantitative measurement of productivity loss due to thermal discomfort, Energ. Buildings 43 (5) (2011) 1057-1062.

[9] N. Romeijn, et al., Sleep, vigilance, and thermosensitivity, Eur. J. Phys. 463 (1) (2012) 169-176.

[10] C. Cajochen, et al., High sensitivity of human melatonin, alertness, thermoregulation, and heart rate to short wavelength light, J. Clin. Endocrinol. Metab. 90 (3) (2005) 1311-1316.

[11] G.W. McEnany, K.A. Lee, Effects of light therapy on sleep, mood, and temperature in women with nonseasonal major depression, Issues Ment. Health Nurs. 26 (7) (2005) 781-794.

[12] S. Aizawa, H. Tokura, The influence of bright light exposure for several hours during daytime on tympanic temperature level at sweating onset, J. Therm. Biol. 23 (2) (1998) 99-106.

[13] M. Ruger, et al., Time-of-day-dependent effects of bright light exposure on human psychophysiology: comparison of daytime and nighttime exposure, Am. J. Phys. Regul. Integr. Comp. Phys. 290 (5) (2006) R1413-R1420.

[14] M. te Kulve, et al., The influence of light on thermal responses, Acta Physiol (Oxford) 216 (2) (2016) 163-185.

[15] T. Roenneberg, M. Merrow, Munich Chronotype Questionnaire, LudwigMaximilians-Universitat, Munchen, 2006Available from: https://www.bioinfo.mpg. $\mathrm{de} / \mathrm{mctq} /$ core_work_life/core/introduction.jsp?language = eng.

[16] W.D. van Marken Lichtenbelt, et al., Evaluation of wireless determination of skin temperature using iButtons, Physiol. Behav. 88 (4-5) (2006) 489-497.

[17] K.C. Parsons, Human Thermal Environments, the Effects of hot, Moderate, and Cold Environments on Human Health, Comfort and Performance, second ed., Taylor \& Francis, 2001.

[18] K. Kaida, et al., Validation of the Karolinska sleepiness scale against performance and EEG variables, Clin. Neurophysiol. 117 (7) (2006) 1574-1581.

[19] D.F. Dinges, A. Powell, Microcomputer analyses of performance on a portable, simple visual RT task during sustained operations, Behav. Res. Methods Instrum. Comput. 17 (6) (1985) 652-655.

[20] C.W. Loh, et al., The validity of psychomotor vigilance tasks of less than 10-minute duration, Behav. Res. Methods 36 (2) (2004) 339-346.

[21] R.J. Lucas, et al., Measuring and using light in the melanopsin age, Trends Neurosci. 37 (1) (2014) 1-9.

[22] N. Santhi, et al., Morning sleep inertia in alertness and performance: effect of cognitive domain and white light conditions, PLoS One 8 (11) (2013) e79688.

[23] P. Badia, et al., Bright light effects on body temperature, alertness, EEG and behavior, Physiol. Behav. 50 (3) (1991) 583-588.

[24] K.C.H.J. Smolders, Y.A.W. de Kort, P.J.M. Cluitmans, A higher illuminance induces alertness even during office hours: findings on subjective measures, task performance and heart rate measures, Physiol. Behav. 107 (1) (2012) 7-16.

[25] V. Gabel, et al., Dawn simulation light impacts on different cognitive domains under sleep restriction, Behav. Brain Res. 281 (2015) 258-266.

[26] E. Rautkyla, et al., Effects of correlated colour temperature and timing of light exposure on daytime alertness in lecture environments, J. Light. Vis. Environ. 34 (2) (2010) 59-68.

[27] M. Hébert, et al., The effects of prior light history on the suppression of melatonin by light in humans, J. Pineal Res. 33 (4) (2002) 198-203.

[28] J. Foret, et al., Effect of morning bright light on body temperature, plasma cortisol and wrist motility measured during 24 hour of constant conditions, Neurosci. Lett. 155 (1993) 155-158.

[29] P. Zhang, H. Tokura, Thermoregulatory responses in humans during exercise after exposure to two different light intensities, Eur. J. Appl. Physiol. 79 (1999) 285-289.

[30] J. Foret, A. Daurat, G. Tirilly, Effect of bright light at night on core temperature, subjective alertness and performance as a function of exposure time, Scand. J. Work Environ. Health 24 (3) (1998) 115-120.

[31] D.J. Dijk, C. Cajochen, A.A. Borbely, Effect of a single 3-hour exposure to bright light on core body temperature and sleep in humans, Neurosci. Lett. 121 (1-2) (1991) 59-62.

[32] K. Ishibashi, et al., Thermoregulatory effect in humans of suppressed endogenous melatonin by pre-sleep bright-light exposure in a cold environment, Chronobiol. Int. 27 (4) (2010) 782-806.

[33] A. Ishimoto, et al., Physiological significance of 3-h bright and dim light exposure prior to taking a bath for core and forehead skin temperatures and heart rate during 1-h bathing of $38.5^{\circ} \mathrm{C}$, J. Therm. Biol. 23 (6) (1998) 353-357.

[34] H.E. Kim, H. Tokura, Influence of light intensities on dressing behavior in elderly people, J. Physiol. Anthropol. Appl. Hum. Sci. 19 (2000) 13-19.

[35] A.J. Lewy, et al., Light suppresses melatonin secretion in humans, Science 210 (4475) (1980) 1267-1269.

[36] N. Santhi, et al., The spectral composition of evening light and individual differences in the suppression of melatonin and delay of sleep in humans, J. Pineal Res. 53 (1) (2012) 47-59.

[37] K. Krauchi, et al., Thermoregulatory effects of melatonin in relation to sleepiness, Chronobiol. Int. 23 (1-2) (2006) 475-484.

[38] C. Cajochen, et al., EEG and ocular correlates of circadian melatonin phase and human performance decrements during sleep loss, Am. J. Phys. 277 (1999) R640-R649.

[39] C.M. Jung, et al., Acute effects of bright light exposure on cortisol levels, J. Biol Rhythm. 25 (3) (2010) 208-216.

[40] F.A. Scheer, R.M. Buijs, Light affects morning salivary cortisol in humans, J. Clin. Endocrinol. Metab. 84 (9) (1999) 3395-3398.

[41] A.C. Guyton, J.E. Hall, Chapter 60: the autonomic nervous system and the adrenal medulla, Textbook of Medical Physiology, Elsevier Inc., 2007, pp. 748-759.

[42] Guyton, A.C. and J.E. Hall, Textbook of Medical Physiology, Chapter 73: body temperature, temperature regulation, and fever. 2007, Elsevier Inc. p. 889-901.

[43] K. Krauchi, et al., Functional link between distal vasodilation and sleep onset latency? Am. J. Phys. Regul. Integr. Comp. Phys. 278 (2000) R741-R748.

[44] K. Krauchi, et al., Warm feet promote the rapid onset of sleep, Nature 401 (6748) (1999) 36-37.

[45] K. Wright, J.T. Hull, C. Czeisler, Relationship between alertness, performance and body temperature in humans, Am. J. Phys. Regul. Integr. Comp. Phys. 283 (2002) R1370-R1377. 